\title{
Le point \\ sur la stratification esthétique des composites
}

\section{RÉSUMÉ}

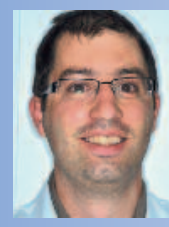

Nicolas DECERLE

CHU de Clermont-Ferrand,

Service d'Odontologie.

Université d'Auvergne Clermond-Ferrand I,

Faculté de Chirurgie Dentaire.

Centre de recherche

en Odontologie clinique (EA 3847).

11, boulevard Charles-de-Gaulle,

63000 Clermont-Ferrand.

Yann-Loïg TURPIN

Faculté de Chirurgie Dentaire et Service d'Odontologie de Clermont-Ferrand.

Centre de Recherche

en Odontologie Clinique (EA 3847).

\section{Céline DESA}

Docteur en chirurgie dentaire.

\section{Martine HENNEQUIN}

Faculté de Chirurgie Dentaire et Service d'Odontologie de Clermont-Ferrand.

Centre de Recherche

en Odontologie Clinique (EA 3847).

La stratification esthétique est une technique qui parait souvent difficile aux praticiens. Les premiers schémas de stratification proposés étaient excessivement complexes mais produisaient et produisent des résultats intéressants pour des praticiens expérimentés. Du fait de l'évolution des matériaux, des schémas plus adaptés ont été proposés. Aujourd'hui existe-t-il une technique qui est tout à la fois esthétique, reproductible et rapide ? En s'appuyant sur la littérature cet article décrira les grands types de stratification, leur différences, leurs résultats. Les techniques de stratifications décrites sont les techniques en 2 couches, 3 couches, selon Vanini, et le Natural Layering Concept. Les résultats en termes de facilité d'utilisation, de prise de teinte, d'intégration optiques sont discutés, ainsi que la place des composites au sein du gradient thérapeutique.

- résine composite

esthétique

- restauration dentaire
AOS 2011;256:341-352

DOI: $10.1051 /$ aos/2011407

(C) EDP Sciences 


\section{Introduction}

$>$

Les praticiens s'accordent sur le prestige que peut apporter au cabinet la réussite des restaurations esthétiques directes en secteur antérieur. Cette notion de prestige est confirmée par la littérature [1]. Il apparait donc nécessaire de maîtriser une méthode de stratification des composites, simple et surtout reproductible.

La stratification esthétique est souvent une technique qui paraît difficile aux praticiens. Et il est vrai que les premiers schémas de stratification proposés étaient excessivement complexes car issus des procédés utilisés dans les techniques de céramo-métallique [2-4]. Or il existe de nombreuses différences entre l'utilisation de la céramique et celle du composite, concernant le matériau en lui-même évidemment, les propriétés optiques, les épaisseurs disponibles...

Ces techniques de stratification des composites produisaient et produisent toujours des résultats intéressants pour des praticiens expérimentés. Mais du fait de leurs complexités et de l'évolution des matériaux, des schémas plus adaptés ont été proposés se basant sur une étude des tissus naturels de la dent. Cette technique est communément appelé Natural Layering Concept [5].

Aujourd'hui, existe-t-il une technique à la fois esthétique, reproductible et rapide ? En s'appuyant sur la littérature cet article décrira les grands types de stratification, leur différences, leurs résultats.

Certaines notions sont nécessaires pour aborder ce sujet. La couleur est définie par trois paramètres : la teinte (ou tonalité chromatique), la saturation et la luminosité. Pour l'évaluation des qualités esthétiques d'une dent comme celles des matériaux de restaura- tion, il est nécessaire de prendre en compte en plus : la translucidité, la fluorescence et l'opalescence.

La teinte ou tonalité chromatique est la qualité par laquelle nous distinguons une famille de couleur d'une autre (vert, bleu, rouge,...) (hue en anglais).

La saturation est la qualité par laquelle nous distinguons une couleur vive d'une couleur pâle, c'est la quantité de pigment contenue dans une couleur (value en anglais)

La luminosité est la qualité par laquelle nous distinguons une couleur claire d'une couleur sombre. Le noir a une luminosité nulle et le blanc a une luminosité maximum (brightness en anglais) [6].

Pour la plupart des praticiens la teinte semble être l'élément le plus important concernant la réussite esthétique. Différents auteurs ont démontré que le facteur prépondérant est en fait la luminosité. Elle n'est pas constante au niveau d'une même dent : elle est faible au niveau du bord incisif, forte au niveau du tiers moyen et moyenne au niveau du tiers cervical. Elle est intimement corrélée à l'état de surface [7].

Globalement la couleur de la dent est principalement déterminée par celle de la dentine qui présente une opacité plus importante que l'émail. La luminosité est quant à elle, dépendante de l'émail qui diminue la saturation de la dentine [8]. Les caractéristiques optiques de l'émail évoluent avec l'âge, elles ont été décrites par Ubassy en 1993. L'émail d'un patient jeune a une teinte blanche, une forte opalescence, et peu de translucidité. Chez un patient adulte, l'émail a une teinte neutre, moins d'opalescence et une translucidité inter- 
médiaire. Chez un patient âgé, l'émail a une teinte jaune et est très translucide.

Nous voyons que d'autres éléments sont à prendre en compte comme la translucidité, l'opalescence et la fluorescence.

Pour définir la translucidité, il faut définir aussi I'opacité et la transparence. Un matériau est transparent s'il permet le passage de rayons lumineux. II est opaque s'il ne permet pas le passage des rayons lumineux, on ne peut alors rien percevoir à travers celui-ci. S'il est translucide, il laisse également passer les rayons lumineux mais il diffuse la plupart de ces rayons: on peut apercevoir un objet à travers mais on ne peut pas le distinguer parfaitement. La translucidité est très présente sur les dents jeunes au niveau du bord incisif.

L'opalescence est la propriété d'un matériau à apparaître orange-rouge en lumière transmise et bleuté en lumière réfléchie. C'est ce qui donne des reflets bleutés au niveau des bords

\section{Matériel et méthode}

Pour permettre de répondre aux objectifs de cet article une recherche bibliographique a été réalisée par informatique sur le site Medline, basée sur I'utilisation de mots clés : « composite ", " stratification ", " esthetic ", "layer ", " incisoproximal restoration ", " color ». Une sélection a ensuite été faite sur les titres puis le résumé. Un deuxième tri a été fait sur les articles entiers. De plus cette sélection a été augmentée par recherche ascendante.

De très nombreux articles ont été publiés pour décrire la stratification. Derrière ce terme générique se cache de nombreuses méthodes différentes.

Dietchi en 2001 donne une classification et une description précises des principales techniques incisifs des dents jeunes, alors que la teinte même de la dent reste dans le jaune.

La fluorescence est la capacité pour un matériau à absorber une énergie rayonnante et à l'émettre ensuite sous la forme d'une longueur d'onde différente [7]. C'est ce qui arrive lorsqu'une source de lumière proche de l'UV éclaire une dent et la fait paraître plus lumineuse. La dent a une fluorescence naturelle qui n'a pas toujours été reproduite par les différents matériaux de restauration.

Au sein d'une restauration composite, ces différentes propriétés optiques sont reproduites par le matériau lui-même [9] mais surtout par la disposition des différentes couches de composites qui ont des propriétés optiques différentes. II existe par exemple des composites translucides avec une forte opalescence, comme le composite Opal dans le système Empress Direct ${ }^{\circledR}$ chez Ivoclar, qui ne seront utilisés qu'en faible quantité chez des patients jeunes.

de stratification [10]. II nous semble devoir y rajouter la technique décrite par Vanini en 1996 [11], correspondant au système de composite HFO de micérium qui est un hybride entre deux techniques décrites par Dietchi.

\section{Technique historique en deux couches (fig. 1)}

Une première couche est réalisée avec un composite ayant les propriétés esthétiques globales de la dent puis un composite incisal ou transparent vient ensuite mimer le bord incisif. La réussite esthétique de ce type de restauration est basée sur les propriétés de mimétisme du composite utilisé en teinte unique. Ce type de 
restauration présente comme plus gros défaut d'avoir un effet grisé [10].

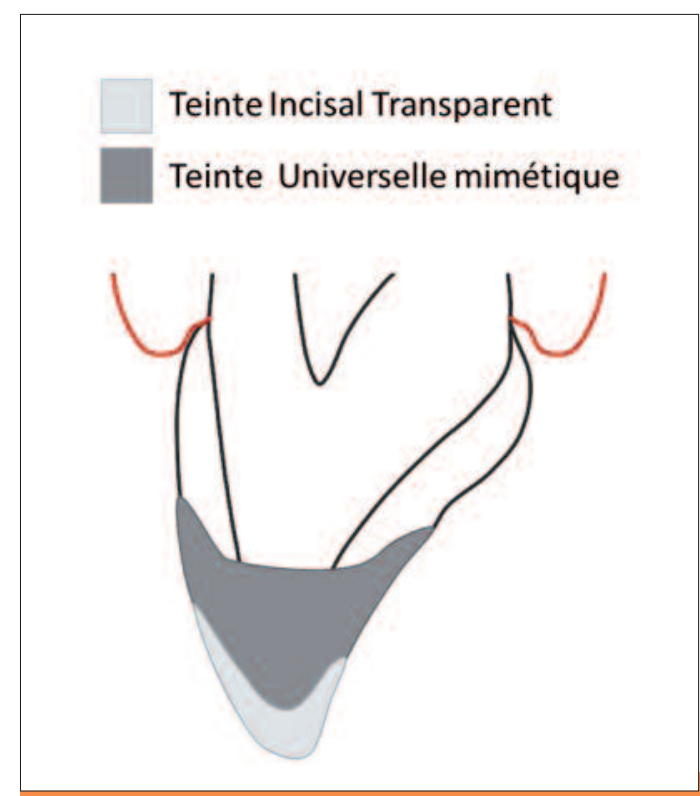

Fig. 1 Technique en 2 couches « historique».

\section{Technique historique}

en trois couches

ou technique

avec deux dentines

d'opacité différentes

ou double effect la yer [12] (fig. 2)

C'est une technique en trois couches utilisant une dentine opaque, une dentine de corps et un composite émail. Différents écueils existent dans cette technique, ce qui explique son apprentissage long. C'est une technique issue des principes de stratification de la céramique en laboratoire avec un opaque pour cacher ce qui doit l'être. Au laboratoire, l'opaque masque l'armature. Dans le cas d'une restauration directe, il convient de masquer le noir de la cavité buccale et le trait de fracture ou la zone de transition entre la dent et la restauration. Ensuite la dentine recrée la teinte de corps de la dent d'où son nom de dentine de corps, puis une couche recrée l'émail.

La plus grosse difficulté est de doser les épaisseurs des différents matériaux. Si cette technique est mal maîtrisée, on obtient des résultats moins satisfaisants au niveau esthétique qu'avec des techniques en deux couches avec un composite « mimétique ».

Une difficulté supplémentaire est la non-standardisation des termes. Selon les fabricants la couche opaque est appelée dentine opaque ou dentine, la couche de dentine de corps est appelée dentine de corps (body) ou dentine, voire émail, et la dernière couche est appelée émail, transparent ou incisal. Dans certaines marques, il existe des teintes de composite pour des dentines opaques, des dentines de corps, des composites émail, des transparents et des transparents opalescents. Tout cela explique les difficultés pour le praticien de comprendre les principes de ce type de stratification, et surtout de les mettre en œuvre rapidement de manière efficace. La courbe d'apprentissage longue a longtemps découragé les praticiens par les

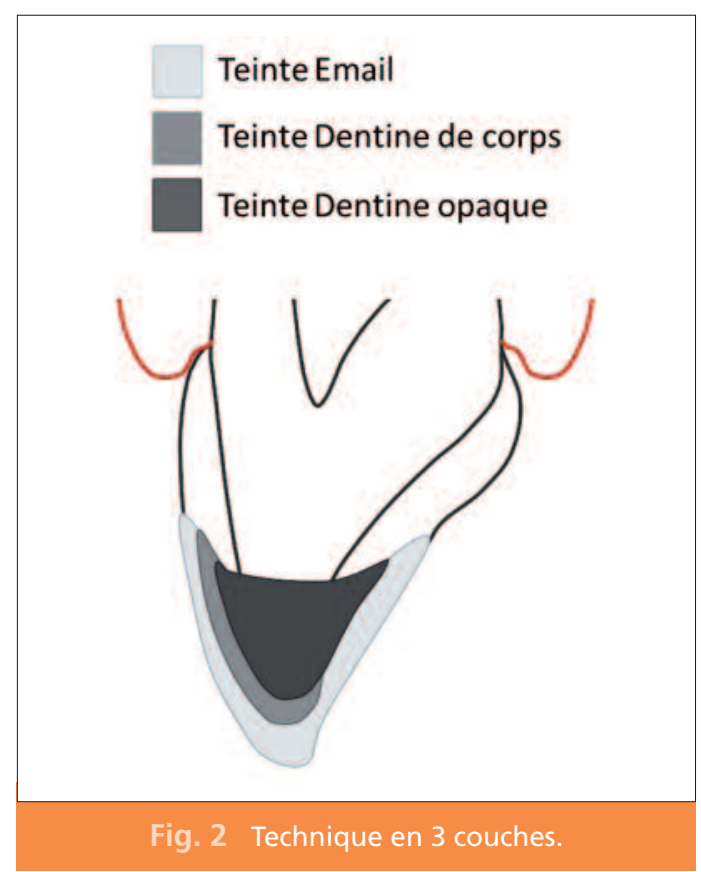


résultats non satisfaisants et non reproductibles ce qui les incitait à revenir à une technique plus simple [13].

Dans cette technique l'épaisseur de la couche d'émail est très faible. Si elle est trop importante, plus d' $1 \mathrm{~mm}$, elle entraine un effet gris. Le composite émail est donc placé en couche d'épaisseur plus faible que la couche d'émail naturel correspondant.

\section{Technique en trois couches selon Vanini \\ avec utilisation \\ de l'émail HFO (fig. 3) \\ ou HRI (fig. 4)}

C'est une technique proche de la technique trois couches qui a été proposée en 1996 [11]. La différence principale entre ces deux techniques, réside dans une couche supplémentaire de résine adhésive utilisée entre la dentine et l'émail. Elle a pour but de mimer la couche

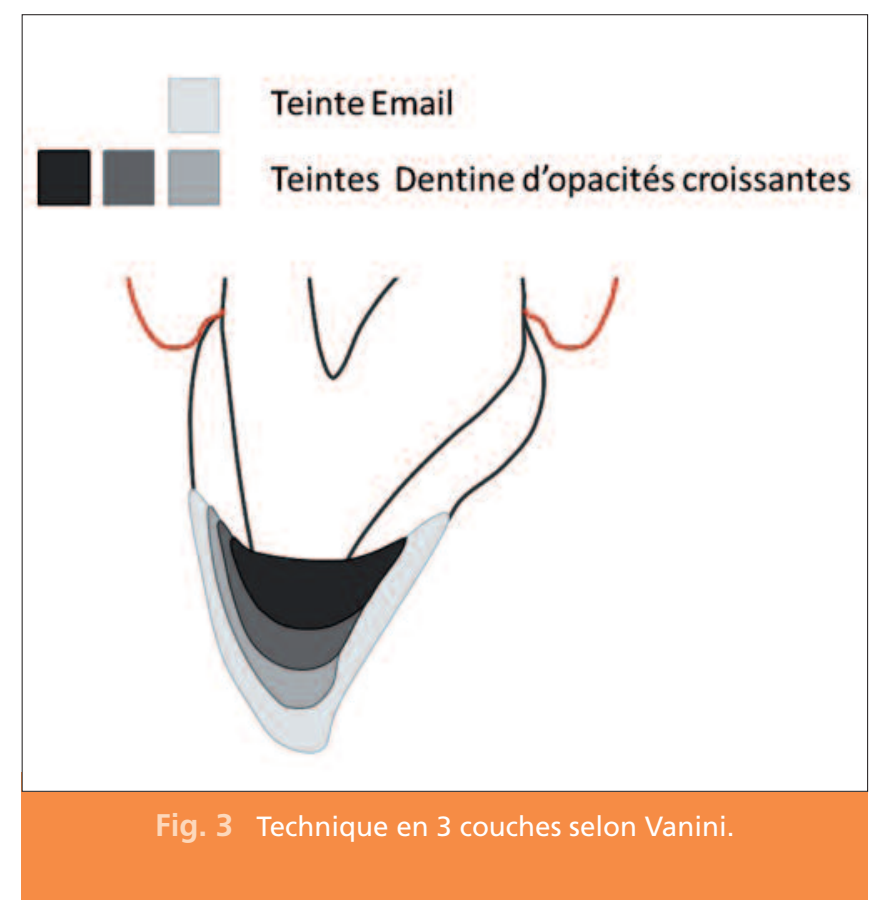

d'émail amorphe et de haute teneur protéique appelée par Vanini couche de haute diffusion ou couche vitreuse. Ici une ou deux saturations de dentines ne sont pas suffisantes pour mimer complètement la dentine. De ce fait dans les restaurations volumineuses Vanini décrit l'utilisation de trois dentines différentes appliquées en couches obliques. II est important de noter que les apports obliques, en plus de favoriser I'esthétique, améliore les forces de contraction au sein du composite [14]. Si la restauration est de plus petit volume, le nombre de dentines différentes diminue.

A la différence des techniques classique en trois couches, la teinte n'est pas définie par le teintier Vita mais dans un teintier particulier où les dentines sont appelées UD1 à UD6. La dentine opaque est remplacée par une dentine ayant deux teintes de plus que celle de corps. Par exemple, pour une restauration en A3 (UD3), la dentine la plus palatine sera une dentine UD5. Dans ce cas si la restauration est de gros volume, il y aura une dentine UD5, UD4, et de

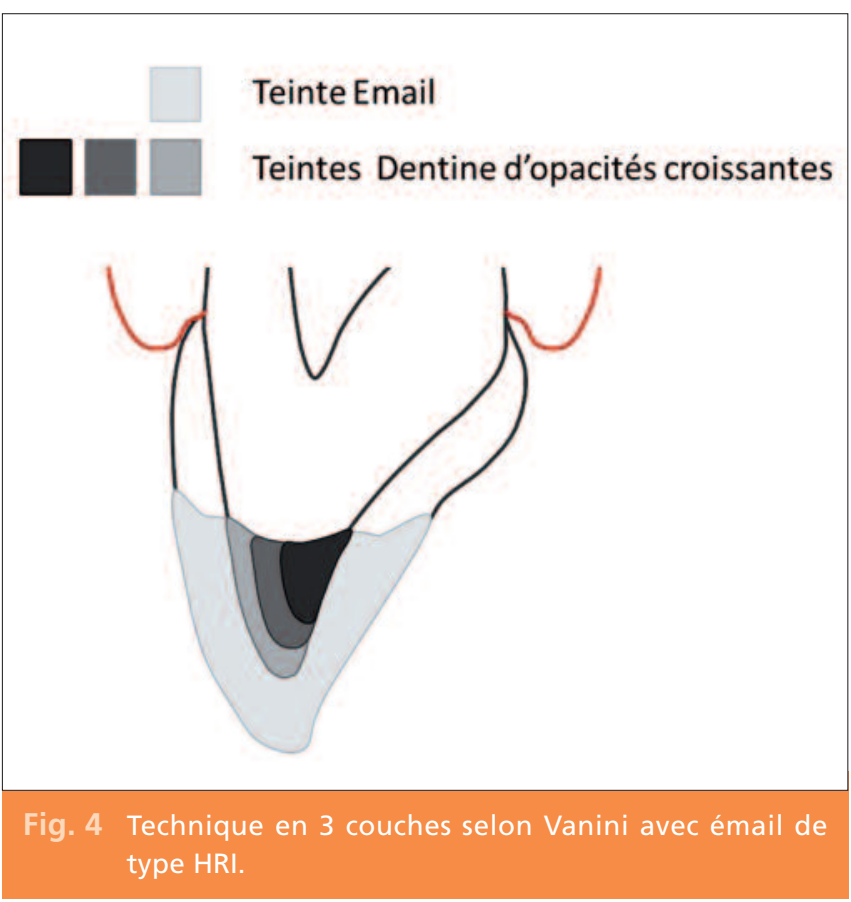


la dentine UD3 : UD5 et UD3 si la restauration est de volume plus petit, et si le volume est faible uniquement de la dentine UD3.

Dans cette technique, si l'émail est celui du système HFO, il doit être placé en couche plus mince que celui qu'il remplace : si c'est celui du système HRI il doit être utilisé en couche équivalente car le coefficient de réfraction du composite HRI est équivalent à celui de l'émail [15] (fig. 4).

\section{Technique du}

\section{Natural Layering Concept}

(fig. 5)

Cette technique a été proposée par Dietchi [5] pour résoudre les difficultés de la technique à trois couches. Dans cette technique de stratification les tissus dentaires sont remplacés par des composites ayant les mêmes principes optiques que les tissus qu'ils remplacent. II n'y a donc que deux composites, un pour la dentine et un pour l'émail. Ils sont placés en couche de la même épaisseur que les tissus qu'ils remplacent, à la

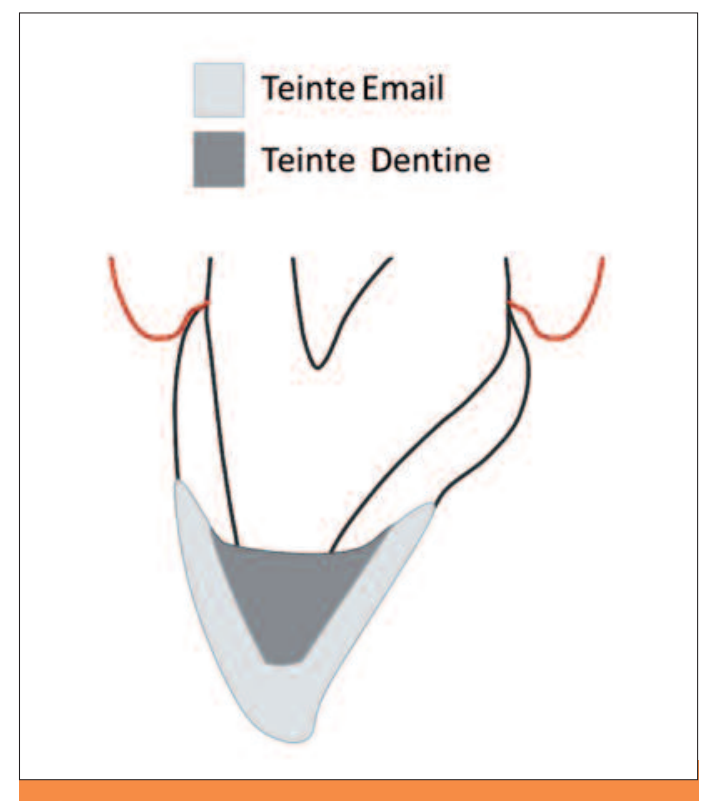

Fig. 5 Technique du « Natural Layering Concept ». seule différence que la dentine recouvre une partie du biseau amélaire pour cacher la transition entre la restauration et la dent.

\section{Technique évoluée du «Natural Layering Concept »}

Cette technique plus ambitieuse est une évolution de la technique du Natural Layering Concept, avec l'ajout d'effets pour reproduire les détails anatomiques les plus fins. Ces matériaux sont le plus souvent ajoutés entre les couches de dentine et d'émail. Les intensifs les plus fréquemment utilisés sont le bleu pour l'opalescence, le doré pour augmenter la saturation dans des zones particulières, et le blanc pour reproduire les plages d'hypominéralisation [16].

Si des composites sont encore commercialisés pour la technique de stratification historique de deux couches, on considère aujourd'hui que ce n'est pas une technique de stratification qui permet d'obtenir des résultats esthétiques suffisants. Les techniques permettant un résultat esthétique suffisant sont donc les techniques à trois couches, ou apparentées comme la technique de Vanini, ou les techniques apparentées au Natural Layering Concept.

Ces deux familles sont représentés par différentes manufactures voire par différents composites au sein d'une même marque. Il est très important de comprendre pour quelle type de stratification le composite est prévu pour permettre une utilisation optimale de celui-ci.

À ces concepts différents, est associée une prise en charge de la restauration différente depuis la prise de teinte jusqu'à la mise en place de la dernière couche de composite. Pour permettre une comparaison simple, nous comparerons la technique trois couches (T3C) à la technique apparentée au Natural Layering Concept (NLC). 


\section{Prise de teinte}

La plupart du temps dans la T3C, le choix de teinte se fera grâce un teintier de type Vita, avec un nombre de teintes important pouvant aller jusqu'à 35 ou 40. Dans le cas du NLC, le teintier Vita est abandonné le plus souvent au profit d'un teintier spécifique ne comportant qu'une seule teinte pour la dentine, généralement nommé $D$, avec des saturations différentes, dont le nombre peut varier dans ces systèmes de 5 à 8 . Concernant l'émail, il est en général limité à trois saturations et le plus souvent nommé $\mathrm{E}$. Dans ces systèmes, il existe en plus des effets facultatifs (Miris ${ }^{\circledR}$ ), et/ou des composites fluides permettant de cacher des discolorations (Amarys Voco $\left.{ }^{\circledR}\right)$.

La prise de teinte est compliquée par de nombreux paramètres, la saturation du matériau varie considérablement avec l'épaisseur des couches. Le teintier est réalisé pour la plupart des manufacturiers en résine méthacrylate teintée, voire en céramique pour le teintier Vita et non en composite, qui est le matériau qui sera utilisé. De plus le composite voit sa teinte modifiée lors de la polymérisation [17], et le fait de superposer

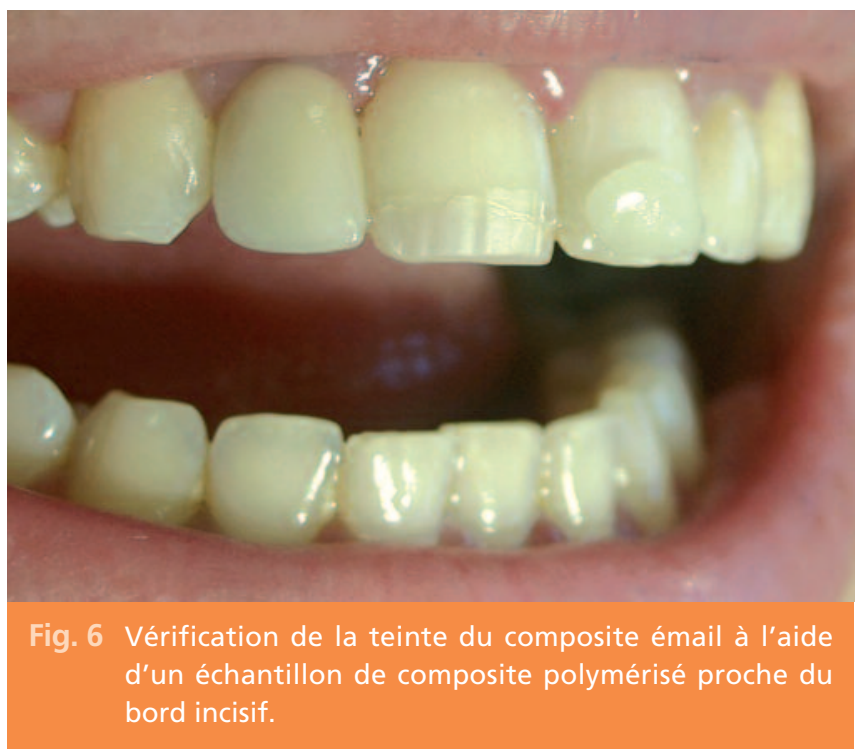

les couches modifie les propriétés optiques des composites [18].

Pour palier ces différents problèmes, Dietchi propose dans son concept Miris ${ }^{\circledR}$, un teintier en composite, avec une possibilité de superposer les échantillons dentine et émail grâce à leur forme, en interposant une couche de glycérine. D'autres auteurs proposent de créer son propre teintier [19] ou de vérifier la prise de teinte à I'aide d'échantillons de composites photopolymérisés directement sur la dent [1] (fig. 6).

Dans les T3C, la prise de teinte est faite soit à I'aide de piges représentant les teintes Vita et la « recette " est associée (Filtek supreme ${ }^{\circledR}$, Esthet $X^{\circledR} \ldots$, soit avec les piges représentant toutes les teintes disponibles dans le système. Ceci rend la prise de teinte extrêmement fastidieuse.

\section{Glé en silicone \\ et première couche palatine}

Les auteurs s'accordent sur l'utilisation d'une clé en silicone pour faciliter la mise en place de la couche la plus palatine ou linguale de composite (fig. 7).

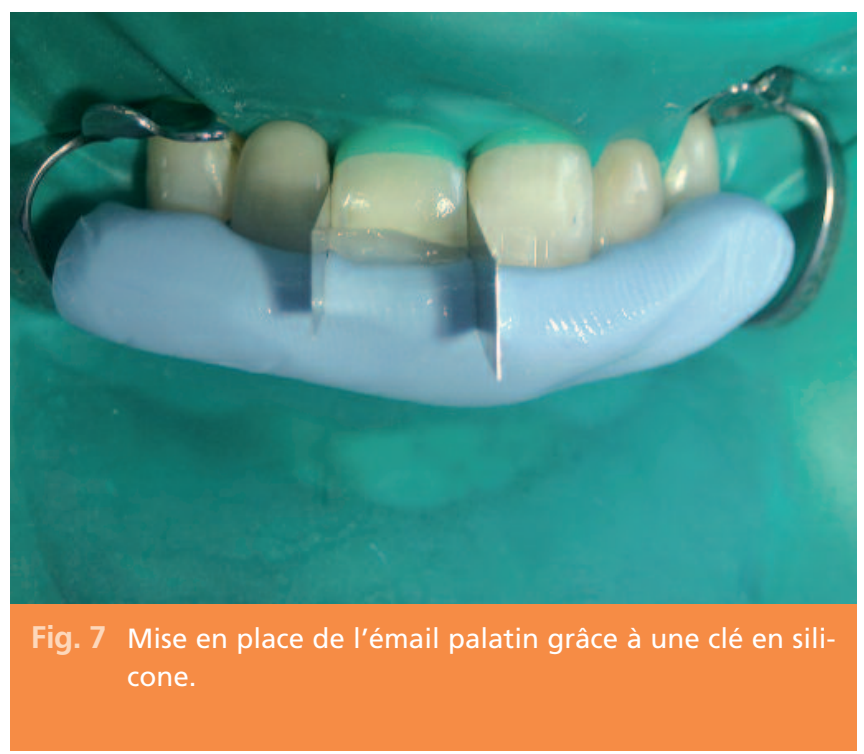


En revanche, si la plupart des auteurs considèrent la mise en place d'une couche d'émail palatine, certains proposent de simplifier la T3C en plaçant directement la dentine opaque au contact de celle-ci [20]. Cela présente l'avantage certain de diminuer le nombre de couche déjà important dans cette technique et de limiter le risque d'épaisseur insuffisante de dentine au profit de l'émail, cause majeure d'échec de cette technique. Cela peut éventuellement permettre aussi de se passer de la clé en silicone, puisque, dans ce cas, la première couche de composite est utilisée en épaisseur suffisante pour avoir une tenue sans I'aide de la clé en silicone ce qui est impossible si le mur d'émail palatin doit avoir une épaisseur de moins d'1 mm (fig. 8).

\section{Mise en place de la dentine}

C'est la principale différence entre les deux types de techniques et elles ont été décrites plus tôt dans cet article. II est à noter que les deux techniques permettent la mise en place d'une anatomie interne de la dentine, par la formation des lobes dentinaires (fig. 9). Une fois cette anatomie réalisée, le positionnement d'effet est possible.

\section{Mise en place d'effets}

Les auteurs proposent cette possibilité dans toutes les techniques, qu'elles soient apparentées à la T3C ou au NLC. L'effet le plus fréquem-

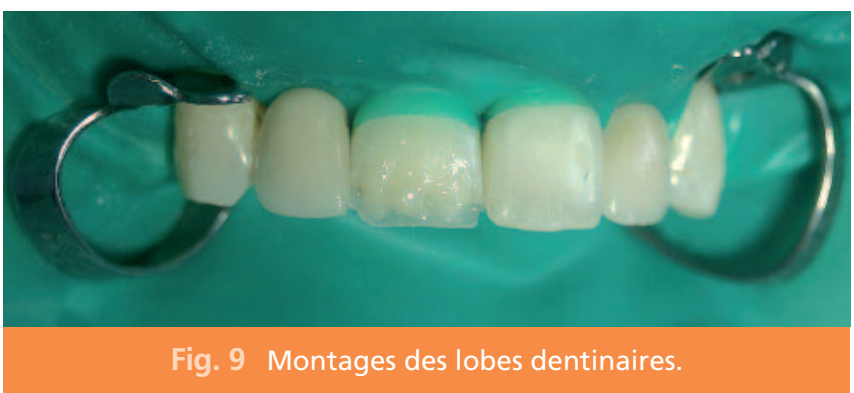

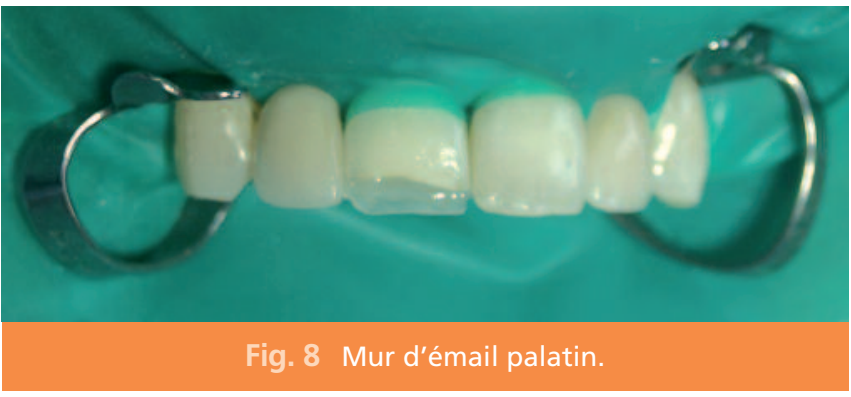

ment utilisé est la mise en place d'un matériau opalescent ou particulièrement translucide dans les espaces laissés par les lobes dentinaires.

\section{Dernière couche d'émail}

C'est une différence importante entre les techniques T3C et NLC. L'épaisseur de composite émail est moins importante que l'émail qu'elle remplace dans la T3C, alors qu'elle est équivalente dans la technique NLC. Parmi les techniques apparentées à la T3C, une seule exception notable concerne I'utilisation du composite HRI dans la technique de Vanini où l'épaisseur du composite émail est équivalente à celle de l'émail naturel.

\section{Polissage}

Cette étape est équivalente dans toutes les techniques. La plupart des auteurs préconisent I'utilisation de disque de type pop-on, pour donner

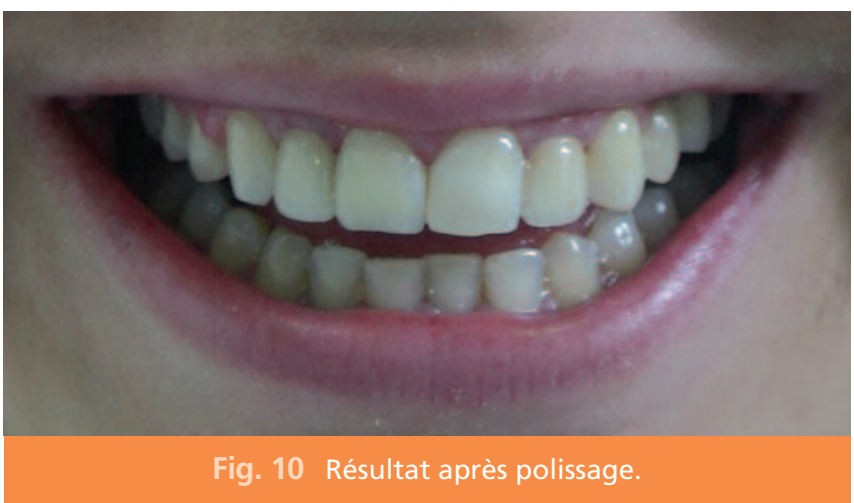


la forme principale, puis par des fraises pour donner la macro- et la microgéographie [21]. Une étude récente [22] montre une supériorité des fraises diamantées par rapport aux fraises

\section{Discussion}

Il existe de nombreux composites sur le marché, et, selon les manufacturiers, tous ces composites devraient permettre une stratification simple et reproductible. Mais comme cela a déjà été dit plus haut, très peu de praticiens utilisent ces techniques, par manque de connaissances des principes d'utilisation. La courbe d'apprentissage longue des techniques plus complexes les défavorise par rapport aux techniques historiques à deux couches. D'autant que l'amélioration des matériaux composites a encore accentué leur mimétisme.

Malgré cela, il existe de nombreuses situations cliniques où ces techniques à deux couches ne permettent pas une restauration satisfaisante, comme dans les cas de restaurations d'angles ou de bords incisifs. Dans ces cas là, faut-il choisir une restauration à trois couches ou apparenté au Natural Layering Concept ?

Il n'existe que peu d'études concernant l'intégration optique des composites suivant le type de stratification et qui pourraient donner un indice scientifique sur le choix de la technique, ou d'une partie de la technique. Une étude récente [24] semble montrer que les restaurations réalisées avec des composites dont le choix de teinte a été fait avec un teintier uni-teinte sont mieux intégrées optiquement que celles réalisées avec un teintier multi-teinte. La difficulté d'analyse de cette étude est que le type de teintier est intimement lié à la philosophie de stratification puisque les composites utilisant multi lames en carbure de tungstène. Une fois ces étapes réalisées, le poli terminal est obtenu grâce à des brossettes et une pâte abrasive [23] (fig. 10).

des techniques à trois couches sont multi-teintes et que ceux utilisant une technique du type NLC sont des teintiers uni-teintes. Malgré tout, cela semble aller dans le sens d'une simplification de l'étape délicate de la prise de teinte.

Une autre manière de faciliter cette étape délicate serait l'utilisation de spectrophotomètres. Mais aujourd'hui cet outil n'a pas encore été décrit pour l'utilisation des composites. Si son utilisation était choisie, elle serait limitée à l'emploi de composites dont le système de référence est le teintier Vita ${ }^{\circledR}$. Or les composites ne présentent qu'une faible correspondance avec ce teintier [25]. D'ailleurs la possibilité de mixer différents composite est quasi impossible du fait de la différence de teinte pour la même désignation entre les différentes marques [26]. Certains auteurs proposent aussi I'utilisation de la photographie et de l'analyse informatique [27].

Une difficulté majeure est que la teinte des composites n'est pas seulement influencée par la composition du matériau, elle est aussi corrélée à son épaisseur [18]. En dessous de 0,5 mm, la teinte est très influencée par les capacités de transmission de la lumière, beaucoup moins audessus de $1 \mathrm{~mm}$ où le facteur le plus important est l'épaisseur elle-même, avec une diminution de la translucidité et une augmentation de I'opalescence avec l'épaisseur [23].

Cela explique que le matériau en lui-même est important mais son utilisation l'est encore plus. 
Donc lorsqu'un praticien utilise un composite, il devra admettre une courbe d'apprentissage pour gérer les épaisseurs qui modifient les capacités optiques.

L'utilisation des composites en stratification a été proposée dans des cas où sont fréquemment choisies des solutions prothétiques comme les facettes ou les couronnes périphériques, par exemple dans les restaurations chez les patients présentant un arrangement inesthétique [13], une usure importante des dents du fait du vieillissement [28] ou de pathologies comme le reflux gastro-œsophagien $[29,30]$. Certains auteurs ont voulu rendre plus objectifs les critères de choix entres les différentes techniques entrant dans des plans de traitement esthétique. Dietchi a proposé une première approche de ces critères de choix et de cette évolution par rapport à la perte de tissu dentaire [31]. Attal et Tirlet ont repris ce concept et l'ont remis en forme dans ce qu'ils appellent le gradient thérapeutique [32]. Les conclusions de ces auteurs sont que la thérapeutique mise en œuvre dans le but de restaurer l'esthétique du sourire doit toujours être la moins invasive possible. Dans cette philosophie, la mise en place de composite est une des thérapeutiques les plus conservatrices de tissus dentaires sains. Elle doit donc être connue et maitrisée pour permettre un choix complet et éclairé des possibilités offertes aux patients, dans le respect du principe de conservation tissulaire qui est majeur aujourd'hui.

Malgré tout, à l'heure actuelle, la littérature est quasi absente sur des critères de choix objectifs permettant de placer le traitement du patient au sein de ce gradient thérapeutique, car il existe encore de nombreuses questions sur l'évolution sur le long terme des composites en stratification. Les traitements par exemple des patients présentant des reflux gastro-œsophagiens, ne sont pour l'instant que des rapports de cas. II n'existe actuellement aucune étude clinique évaluant la qualité de ces restaurations. Ces études sur l'évolution de ces matériaux devront être conduites dans le futur, grâce à des critères d'évaluation récents [33].

\section{Bibliographie}

1. Franco $E B$, Francischone $C E$, Medina-Valdivia JR, Baseggio W.

Reproducing the natural aspects of dental tissues with resin composites in proximoincisal restorations.

Quintessence Int 2007;38(6):505-10.

2. Dietschi D.

Free-hand composite resin restorations: a key to anterior aesthetics. Pract Periodontics Aesthet Dent 1995;7(7):15-25; quiz 27.
3. Magne $\mathrm{P}, \mathrm{Holz} \mathrm{J}$. Stratification of composite restorations: systematic and durable replication of natural aesthetics. Pract Periodontics Aesthet Dent 1996;8(1):61-8; quiz 70.

4. Portalier L. Stratification naturelle des composites antérieurs d'une méthode nouvelle et de son application... Réal Clin 1998;9(3):315-27.

5. Dietschi D. Free-hand bonding in the esthetic treatment of anterior teeth: creating the illusion.

J Esthet Dent 1997;9(4):156-64.

6. Ubassy G. Shape and color : the key to successful ceramic restorations. Chicago: Quintessence Pub Co, 1993.

7. Magne $P$. Bonded porcelain restorations in the anterior dentition. Chicago:Berlin;Paris [etc]: Quintessence Publ 2002. 
8. Baratieri LN, Araujo E, Monteiro S Jr.

Color in natural teeth and direct resin composite restorations: essential aspects.

Eur J Esthet Dent 2007;2(2):172-86.

9. $Y u$ B, Lee $Y-K$. Difference in opalescence of restorative materials by the illuminant.

Dent Mater 2009;25(8):1014-21.

10. Dietschi D.

Layering concepts in anterior composite restorations. J Adhes Dent 2001;3(1):71-80.

11. Vanini $L$.

Light and color in anterior composite restorations. Pract Periodontics Aesthet Dent 1996;8(7):673-82; quiz 684 .

12. Felippe LA, Monteiro S Jr, De Andrada CAC, Ritter AV. Clinical strategies for success in proximoincisal composite restorations. Part II: Composite application technique. J Esthet Restor Dent 2005;17(1):11-21.

13. Devoto $W$, Saracinelli $M$, Manauta J.

Composite in everyday practice: how to choose the right material and simplify application techniques in the anterior teeth. Eur J Esthet Dent 2010;5(1):102-24.

14. Nikolaenko SA, Lohbauer U, Roggendorf M, Petschelt A, Dasch W, Frankenberger R. Influence of c-factor and layering technique on microtensile bond strength to dentin.

Dent Mater

2004;20(6):579-85.

15. Weisrock G, Merz R, Ortet $S$, Koubi S, Tassery $\mathrm{H}$,

Faucher A.

Clonage artificiel de l'émail.

À propos d'un nouveau

composite.

Inf Dent

2009;35:2020-6.

16. Dietschi D, Ardu S, Krejci I.

A new shading concept based on natural tooth color applied to direct composite restorations.

Quintessence Int 2006;37(2):91-102.

17. Çelık EU, Aladağ $A$, Türkün LŞ, Yilmaz G. Color changes of dental resin composites before and after polymerization and storage in water. J Esthet Restor Dent 2011;23(3):179-88.

18. Vichi A, Fraioli $A$, Davidson $\mathrm{CL}$, Ferrari $\mathrm{M}$. Influence of thickness on color in multi-layering technique.

Dent Mater 2007;23(12):1584-9.

19. Østervemb N, Jørgensen JN, Hørsted-Bindslev P. Shade guide optimizationa novel shade arrangement principle for both ceramic and composite shade guides when identifying composite test objects.

J Esthet Restor Dent 2011;23(1):22-32.

20. Kuhn G, Colon P. Composites antérieurs : technique de stratification simplifiée.

Réa Clin 2003;14(4):409-21.

21. Fahl $\mathrm{N} J \mathrm{~J}$.

A polychromatic composite layering approach for solving a complex

Class IV/direct veneer/

diastema combination:

Part II.

Pract Proced Aesthet Dent 2007;19(1):17-22.

22. Maresca C, Pimenta LAF, Heymann HO, Ziemiecki TL, Ritter AV.

Effect of finishing instrumentation on the marginal integrity of resin-based composite restorations.

J Esthet Restor Dent 2010;22(2):104-12.

23. Arimoto $A$, Nakajima $M$, Hosaka K, Nishimura K, Ikeda M, Foxton RM, et al. Translucency, opalescence and light transmission characteristics of light-cured resin composites.

Dent Mater 2010;26(11):1090-7.

24. Magne $P$, So WS. Optical integration of incisoproximal restorations using the natural layering concept.

Quintessence Int 2008 sept;39(8):633-43.

25. da Costa J, Fox P, Ferracane J.

Comparison of various resin composite shades and layering technique with a shade guide. J Esthet Restor Dent 2010;22(2):114-24.

26. Lee $Y-K, Y u B$, Lee $S-H$, Cho M-S, Lee C-Y, Lim H-N. Shade compatibility of esthetic restorative materials - A review. Dent Mater 2010;26(12):1119-26.

27. Milnar FJ. Selecting nanotechnologybased composites using colorimetric and visual 
analysis for the restoration of anterior dentition: a case report. J Esthet Restor Dent 2004;16(2):89-100; discussion 101.

28. Pontons-Melo JC, Furuse AY, Mondelli J.

A direct composite resin stratification technique for restoration of the smile.

Quintessence Int 2011;42(3):205-11.

29. Reis $A$, Higashi $C$, Loguercio AD. Re-anatomization of anterior eroded teeth by stratification with direct composite resin. J Esthet Restor Dent 2009;21(5):304-16.

30. Vailati F, Belser UC. Palatal and facial veneers to treat severe dental erosion: a case report following the three-step technique and the sandwich approach. Eur J Esthet Dent 2011;6(3):268-78.

31. Dietschi D.

Optimizing smile composition and esthetics with resin composites and other conservative esthetic procedures.
Eur J Esthet Dent 2008;3(1):14-29.

32. Tirlet G, Attal JP. Le gradient thérapeutique : un concept médical pour les traitements esthétiques. Inf Dent 2009;91(41/42):2561.

33. Hickel R, Peschke A, Tyas M, Mjör I, Bayne S, Peters M, et al.

FDI World Dental Federation: clinical criteria for the evaluation of direct and indirect restorations-update and clinical examples. Clin Oral Investig 2010;14(4):349-66.

\section{SUMMARY}

\section{Update on aesthetic composite resin layering technique}

Nicolas DECERLE,

Yann-Loïg TURPIN,

Céline DESA,

Martine HENNEQUIN

$$
\begin{aligned}
& \text { Keywords } \\
& \text { - composite resin } \\
& \text { - aesthetic } \\
& \text { - dental restoration }
\end{aligned}
$$

Aesthetic stratification is a technique that seems often difficult for practitioners. The first proposed stratification schemes were overly complex but produced interesting results for experienced practitioners. Because of changing materials, patterns most suitable have been proposed. Today is there a technique that is at once aesthetic, reproducible, and fast? Based on the literature this article decries the major types of stratification, their differences, and their results. Stratification techniques described are the techniques in two layers, three layers, according to Vanini, and "Natural Layering Concept". The results in terms of ease of use, color determination, and optical integration are discussed, as well as the role of composites in the treatment gradient. 